\title{
rTMS for PTSD: Induced merciful oblivion or elimination of abnormal hypermnesia?
}

\author{
Simone Rossi ${ }^{\mathrm{a}, *}$, Stefano F. Cappa ${ }^{\mathrm{b}}$, Monica Ulivellii ${ }^{\mathrm{a}}$, Alberto De Capua ${ }^{\mathrm{c}}$, Sabina Bartalini ${ }^{\mathrm{a}}$ and \\ Paolo M. Rossini ${ }^{\mathrm{d}, \mathrm{e}}$ \\ ${ }^{\mathrm{a}}$ Dipartimento di Neuroscienze, Sezione Neurologia, Università di Siena, Policlinico le Scotte, Viale Bracci, \\ I-53100, Siena, Italy \\ ${ }^{\mathrm{b}}$ Centro di Neuroscienze Cognitive, Università Salute-Vita S. Raffaele, DIBIT Via Olgettina 58, I-20132, Milano, \\ Italy \\ ${ }^{\mathrm{c}}$ Dipartimento di Neuroscienze, Sezione Psichiatria, Università di Siena, Policlinico le Scotte, Viale Bracci, \\ I-53100, Siena, Italy \\ ${ }^{\mathrm{d}}$ AFaR.- Dipartimento di Neuroscienze, S. Giovanni Calibita Fatebenefratelli, Isola Tiberina, I-00186, Roma, Italy \\ ${ }^{\mathrm{e}}$ Clinica Neurologica, Università Campus Biomedico, Via Longoni 71, Roma, Italy
}

\begin{abstract}
Neuroimaging studies and experimental data suggest that symptoms of posttraumatic stress disorder (PTSD) are associated with dysfunctions of neural circuits linking prefrontal cortex and the limbic system that have a role in autobiographic episodic memory. High-frequency repetitive transcranial magnetic stimulation (rTMS) of the right dorsolateral prefrontal cortex (DLPFC) has been suggested to be beneficial to patients with PTSD, transiently alleviating re-experiencing as well as avoidance reactions and associated anxiety symptoms. In healthy humans, converging evidence suggests that rTMS of the right DLPFC interferes with episodic memory retrieval. Hence, we hypothesize that daily applications of rTMS in PTSD patients may reduce access to the set of autobiographical stored events, that, if re-experienced, may cause the overt PTSD symptoms.
\end{abstract}

Keywords: PTSD, TMS, rTMS, amnesia, episodic memory

\section{The concept of autobiographic episodic memory}

Seminal, personally experienced, events occurring along our life-span are stored somewhere in the brain. They can be intentionally retrieved, or can emerge unexpectedly, in each moment of everyday life. Normally, these stored events can give rise to joy or sadness, or even anxiety: this is what is referred as "autonoetic consciousness" [13,37]. Such autobiographic traces are the building blocks of episodic memory, a concept indicating a complex set of human cognitive processes that allows the encoding, storage, and in-

*Corresponding author: Simone Rossi, MD, PhD, Dipartimento di Neuroscienze, Sezione Neurologia, Brain Stimulation and Evoked Potentials Lab, Università di Siena, Policlinico Le Scotte, Viale Bracci, I-53100, Italy. Tel.: +39 0577 585401; Fax: +39 0577 270260; E-mail: Rossisimo@unisi.it. tentional recollection (retrieval) of unique events associated with the context in which they occurred [1]. Therefore, the correct functioning of episodic memory mechanisms is pivotal to form the conscious story of our existence [38]. Traditional neuropsychology has looked at amnesia as the most typical dysfunction of the episodic memory system [36].

\section{Post-traumatic stress disorder (PTSD) as a dysfunction of autobiographic memory}

The intrusive, sometimes iterative, recollection of personally experienced unpleasant past events may cause, in some people, a disabling clinical syndrome known as posttraumatic stress disorder (PTSD), characterized by emotional numbing and withdrawal, psychological and physiological hyperarousal, as stated in 
the DSM-IV. Depression, anxiety and avoidance reactions may accompany the re-experiencing of such autobiographical target events. Functional neuroimaging and neurophysiological techniques have identified significant neurobiologic changes in the brain of people suffering from PTSD, and these changes take place in areas known to be important in both stress and memory (see [24]). Such advances in cognitive neuroscience have begun to unveil intriguing relationships between memory disturbances and affective disorders (see [17]) both in vivo and at experimental levels. By measuring brain regional cerebral blood flow or metabolism, it has been suggested that dysfunctions of neural circuits linking prefrontal cortex, anterior-cingulate, amygdala and limbic system are detectable in PTSD patients [7,35], together with hyperexcitability at cortical levels, probably due to dysfunction of cortico-cortical inhibitory networks [6]. These functional abnormalities are even more apparent using symptom provocation paradigms, during which dysfunctions of prefrontal cortex and limbic system are associated with the retrieval of the target event or with the overt PTSD syndrome [2,19,35].

Experimentally derived data indicates that acute stress affects brain activity by promoting neuroplastic long-term changes of synaptic efficacy sustained by long-term potentiation (LTP) of excitatory synapses. LTP, which refers to the enduring facilitation of the communication between two neurons following the activation of the synapses by which they are interconnected, is believed to be the substrate for both explicit and implicit learning and memory processes (see [14, 22]). Environmental, physical, and mental stressful events are factors able to promote LTP changes [15] taking place in brain structures and systems that are relevant for both memory processing and the stress response [3]. Hence, it can be postulated that such neuroplastic changes could represent the synaptic correlates of the maintenance of traumatic autobiographic memories that cause PTSD [6].

\section{PTSD can be alleviated by repetitive transcranial magnetic stimulation (rTMS)}

In a recent double-blind, placebo-controlled study that appeared in the American Journal of Psychiatry, Cohen and coworkers [8] applied, in a cohort of PTSD patients, trains of high-frequency $(10 \mathrm{~Hz})$ rTMS for 10 days over two weeks. TMS is currently the only technique that can non-invasively produce a transient dysfunction of the targeted cortical area $[25,41]$. In the Co- hen et al. study, the targeted brain region was the right dorsolateral prefrontal cortex (DLPFC). They found a significant, relatively long-lasting, behavioral improvement regarding both re-experiencing and avoidance, together with alleviation of anxiety symptoms.

The results of the Cohen et al. study [8], which probably opens new neuromodulatory treatment avenues for PTSD, have been interpreted according to the hypothesis of rTMS-induced long-lasting activation of the hypofunctioning right DLPFC. The authors hypothesized that this "activation" would be associated with improvements of the hypofunctionig hypothalamic-pituitaryadrenal axis, with enhanced activity of the depressor area of autonomic responsiveness, or with an increased functional suppression of the amygdala. A further speculation was that high-frequency rTMS of the right DLPFC might have improved functional connectivity mechanisms in PTSD patients. These interesting conjectures are certainly worthy of consideration: Future studies should be aimed to evaluate in patients with PTSD treated by daily rTMS changes in hormonal blood levels, autonomic function, and functional coupling of regional electroencephalographic cortical rhythmicity.

\section{4. rTMS and episodic memory}

Another hypothesis, not mutually exclusive with that from the Cohen et al. study, merges recent evidence from episodic memory studies with PTSD pathophysiology to account for rTMS-induced beneficial effects. To better explain this novel hypothesis in the following paragraph, recent data on TMS and episodic memory should be briefly discussed.

First, we should consider that, although mechanisms of action of high-frequency rTMS are not yet fully understood, it is quite clear that transient interference of brain function is more likely to occur than behavioral improvements $[27,41]$. Of note, the term "virtual lesion" has been coined to depict rTMS effects on behavior [25]. Recent data also suggest that high-frequency rTMS may promote neuroplastic LTPmediated changes at cortical levels [10].

On the basis of this "interferential" approach, highfrequency rTMS $(20 \mathrm{~Hz})$ applied on the right DLPFC (i.e., the same target region of the PTSD study) in healthy humans has been used to prove the existence of a causal relationship between this brain region and retrieval processes during episodic memory tasks: Under rTMS, but not during sham stimulation, subjects made 
significant recognition errors of previously encoded visual scenes [28,29] or verbal items [12,33], thus demonstrating that the correct functioning of episodic memory system actually relies on PFC function, with a clear-cut right functional prevalence for retrieval mechanisms of complex visuospatial pictures representing real-life environments $[28,29]$. These findings are in line and extend previous correlational results of many neuroimaging studies based on regional changes of cerebral blood flow and metabolism $[4,5,11]$. While these results have been collected in experimental studies dealing with recently memorized information, there is now ample evidence that the prefrontal cortex is engaged also by the retrieval of remote autobiographical memories [26], the types of memories that are probably dysfunctional in PTSD.

\section{An alternative hypothesis to explain rTMS effects in PTSD}

For a single and brief train of stimulation, as would be applied in memory studies $[12,28,29,33]$, the interferential effect is obviously short-lived, lasting a matter of seconds (i.e., sufficiently long for the subject to answer after the elaboration of the presented visual stimulus). In patients with PTSD, daily applications of high-frequency rTMS to the right DLPFC (which, as previously described, is functionally-necessary for autobiographical retrieval mechanisms) might have induced, probably through temporal summation, a prolonged dysfunction of the brain mechanisms underlying the episodic retrieval for personally experienced real-life events (i.e., the autonoetic consciousness).

Interestingly, patients with PTSD are very suitable candidates for rTMS interference during long-term memory tasks, due to their functional hypoactivation of the right DLPFC [2,19] and to the relatively frequent association of other memory dysfunctions with this psychiatric syndrome $[16,40]$. Assuming that LTP in brain circuitries relevant for recall represents the synaptic correlates of the maintenance of traumatic autobiographic memories that cause PTSD [6], it can be hypothesized that rTMS of the DLPFC interferes with such mechanisms. Therefore, daily interference with rTMS may produce a sort of amnesia for the distressing life event that is the prime mover for PTSD.

Looking at PTSD symptoms from a different perspective, but still in the frame of a dysfunction of neural circuitry underlying memory processes, the "abnormal" autobiographic retrieval might be even regarded as sharing behavioral similarities with the automatic hypermnesia as the one seen in schizophrenic or in autistic savant patients [20,23]. Along this vein, an intriguing hypothesis is that the abnormal and intrusive reiteration of the target autobiographical event might induce - while simultaneously being sustained by- synaptic changes linked with LTP mechanisms. In the longterm, these mechanisms may contribute, together with the partial loss of right DLPFC modulatory activity which is hypofunctioning in PTSD patients, to re-shape paradoxical fronto-temporal-limbic functional connections sustaining PTSD symptoms. Similar mechanisms of aberrant synaptic plasticity are known to take place in other neurological conditions such as stroke or chronic pain [30]. Notably, rTMS may have some transient beneficial effect both in stroke recovery and chronic pain treatment $[18,21]$. Through such a mechanism, daily rTMS may interrupt the intrusive reiteration of the target autobiographical event through a resetting of the DLPFC function.

In other words, whatever the mechanism, daily rTMS may temper to a more physiological level the intrusive, LTP-mediated access to the set of stored events, that, if re-experienced, cause the overt PTSD picture, thereby restoring a better-functioning autonoetic consciousness mechanism. If this memory-oriented hypothesis put forward to explain beneficial effects of rTMS in PTSD is true, then the classical manifestation of an episodic memory dysfunction (i.e., amnesia) could be regarded either as a merciful oblivion or as a sort of downregulation of abnormal hypermnesia processes, resulting in symptom alleviation for these patients.

\section{Questions for future research}

How might these hypotheses be reliably addressed in future TMS research? The best approach is probably to start from experimental data. Fear conditioning is a form of associative learning between certain neutral stimuli (conditioned stimuli) and aversive events (unconditioned stimuli), and is considered to be a suitable animal model of PTSD [39]. A bulk of experimental studies based on the fear conditioning paradigm $[9,31$, $32,34]$ converge to suggest that the acquisition and retrieval of an intense fear, mimicking a traumatic event and its repeated recollection, have an impact on LTP induction in the synapses of the dorsal hippocampus. Such induction may underpin the mechanism of memory deficits by stress or trauma re-experiencing in PTSD patients. Verifying how and how long these synaptic 
changes are modulated by rTMS (and if these changes are paralleled by behavioural improvement toward fear conditioning and response) seems an appropriate "first step" to better understand the effects of rTMS on human PTSD.

\section{References}

[1] A. Baddeley, M. Conway and J. Aggleton, eds, Episodic memory, Phil Trans $R$ Soc London B356 (2001), 1341-1515.

[2] J.D. Bremner, M. Vythilingam, E. Vermetten, S.M. Southwick, T. McGlashan, L.H. Staib, R. Soufer and D.S. Charney. Neural correlates of declarative memory for emotionally valenced words in women with posttraumatic stress disorder related to early childhood sexual abuse, Biol Psychiatry 53 (2003), 879-889.

[3] R.A. Bryant, Early predictors of posttraumatic stress disorder, Biol Psychiatry 53 (2003), 789-795.

[4] R.L. Buckner and M.E. Wheeler, The cognitive neuroscience of remembering, Nat Neurosci Rev 2 (2001), 624-634.

[5] R. Cabeza and L. Nyberg, Imaging Cognition II: an empirical review of 275 PET and fMRI studies, J Cogn Neurosci 12 (2000), 1-47.

[6] D. Centonze, M.G. Palmieri, L. Boffa, M. Pierantozzi, P. Stanzione, L. Brusa, M.G. Marciani, A. Siracusano, G. Bernardi and M.D. Caramia, Cortical hyperexcitability in posttraumatic stress disorder secondary to minor accidental head trauma: a neurophysiologic study, J Psychiatry Neurosci 30 (2005), 127-132.

[7] Y. Chung, S.H. Kim, S.K. Chun, J. Chae, D.W. Yang, H.S. Sohn and J. Jeong, Alterations in cerebral perfusion in posttraumatic stress disorder patients without re-exposure to accident-related stimuli, Clin Neurophysiol 117 (2006), 637642.

[8] H. Cohen, Z. Kaplan, M. Kotler, L. Kouperman, R. Moisa and N. Grisaru, Repetitive transcranial magnetic stimulation of the right dorsolateral prefrontal cortex in posttraumatic stress disorder: a double-blind, placebo-controlled, study, Am J Psychiat 161 (2004), 515-524

[9] V. Doyere, C. Redini-Del Negro, G. Dutrieux, G. Le Floch, S. Davis and S. Laroche, Potentiation or depression of synaptic efficacy in the dentate gyrus is determined by the relationship between the conditioned and unconditioned stimulus in a classical conditioning paradigm in rats, Behav Brain Res $\mathbf{7 0}$ (1995), 15-29.

[10] S.K. Esser, R. Huber, M. Massimini, M.J. Peterson, F. Ferrarelli and G. Tononi, A direct demonstration of cortical LTP in humans: a combined TMS/EEG study, Brain Res Bull 69 (2006), 86-94.

[11] P.C. Fletcher and R.N.A. Henson, Frontal lobes and human memory. Insights from functional neuroimaging, Brain $\mathbf{1 2 4}$ (2001), 849-881

12] A. Floel, D. Poeppel, E.A. Buffalo, A. Braun, C.W. Wu, H.J. Seo, K. Stefan, S. Knecht and L.G. Cohen, Prefrontal cortex asymmetry for memory encoding of words and abstract shapes, Cereb Cortex 14 (2004), 404-409.

[13] J.M. Gardiner, Episodic memory and autonoetic consciousness: a first-person approach, Philos Trans $R$ Soc London B356 (2001), 1351-1361.

[14] E.R. Kandel, The molecular biology of memory storage: a dialogue between genes and synapses, Science 294 (2001), 1030-1038.
[15] J.J. Kim and K.S. Yoon, Stress: metaplastic effects in the hippocampus, Trends Neurosci 21 (1998), 505-509.

16] C.L. Isaac, D. Cushway and G.V. Jones, Is posttraumatic stress disorder associated with specific deficits in episodic memory? Clin Psychol Rev (2006), epub ahead of print.

[17] K. LaBar and R. Cabeza, Cognitive neuroscience of emotional memory, Nat Rev Neurosci 7 (2006), 54-64.

18] J.P. Lefaucheur, Transcranial magnetic stimulation in the management of pain, Suppl Clin Neurophysiol 57 (2004), 737 748.

[19] I. Liberzon, J.C. Britton and K.L. Phan, Neural correlates of traumatic recall in posttraumatic stress disorder, Stress 6 (2003), 151-156.

[20] R.J. Linscott and R.G. Knight, Automatic hypermnesia and impaired recollection in schizophrenia, Neuropsychology 15 (2001), 576-585.

21] C.G. Mansur, F. Fregni, P.S. Boggio, M. Riberto, J. GallucciNeto, C.M. Santos, T. Wagner, S.P. Rigonatti, M.A. Marcolin and A. Pascual-Leone, A sham stimulation-controlled trial of rTMS of the unaffected hemisphere in stroke patients, Neurology 64 (2005), 1802-1804.

[22] S.J. Martin, P.D. Grimwood and R.G.M. Morris, Synaptic plasticity and memory: an evaluation hypothesis, Annu Rev Neurosci 23 (2000), 649-711.

[23] L. Mottron, S. Belleville, E. Stip and K. Morasse, Atypical memory performance in an autistic savant, Memory 6 (1998), 593-607.

24] D.J. Nutt and A.L. Malizia, Structural and functional brain changes in posttraumatic stress disorder, J Clin Psychiatry 65(Suppl. 1) (2004), 11-17.

[25] A. Pascual-Leone, V. Walsh and J.C. Rothwell, Transcranial magnetic stimulation in cognitive neuroscience. Virtual lesion, chronometry and functional connectivity, Curr Opin Neurobiol 10 (2000), 232-237.

[26] M. Piefke, P.H. Weiss, K. Zilles, H.J. Markowitsch and G.R. Fink, Differential remoteness and emotional tone modulate the neural correlates of autobiographical memory, Brain $\mathbf{1 2 6}$ (2003), 650-668.

[27] S. Rossi and P.M. Rossini, TMS in cognitive plasticity and the potential for rehabilitation, Trends Cogn Sci 8 (2004), 273279

[28] S. Rossi, S.F. Cappa, C. Babiloni, P. Pasqualetti, C. Miniussi, F. Carducci, F. Babiloni and P.M. Rossini, Prefrontal cortex in long-term memory: an "interference" approach using magnetic stimulation, Nat Neurosci 4 (2001), 948-952.

[29] S. Rossi, C. Miniussi, P. Pasqualetti, C. Babiloni, P.M. Rossini and S.F. Cappa, Age-related functional changes of prefrontal cortex in long-term memory. A repetitive transcranial magnetic stimulation (rTMS) study, J Neurosci 24 (2004), 79397944.

[30] P.M. Rossini and G. Dal Forno, Neuronal post-stroke plasticity in the adult, Restor Neurol Neurosci 22 (2004), 193-206.

[31] B. Sacchetti, C.A. Lorenzini, E. Baldi, C. Bucherelli, M. Roberto, G. Tassoni and M. Brunelli, Long-lasting hippocampal potentiation and contextual memory consolidation, Eur J Neurosci 13 (2001), 2291-2298.

[32] B. Sacchetti, E. Baldi, C.A. Lorenzini and C. Bucherelli, Differential contribution of some cortical sites to the formation of memory traces supporting fear conditioning, Exp Brain Res 146 (2002), 223-232.

[33] M. Sandrini, S.F. Cappa, S. Rossi, P.M. Rossini and C. Miniussi, The role of prefrontal cortex in verbal episodic memory: rTMS evidence, J Cogn Neurosci 15 (2003), 855-861. 
[34] T. Seidenbecher, T.R. Laxmi, O. Stork and H.C. Pape, Amygdalar and hippocampal theta rhythm synchronization during fear memory retrieval, Science 301 (2003), 846-850.

[35] L.M. Shin, S.M. Kosslyn, R.J. McNally, N.M. Alpert, W.L. Thompson, S.L. Rauch, M.L. Macklin and R.K. Pitman, Visual imagery and perception in posttraumatic stress disorder. A positron emission tomographic investigation, Arch Gen Psychiatry 54 (1997), 233-241.

[36] L.R. Squire, R.E. Clark and B.J. Knowlton, Retrograde amnesia, Hippocampus 11 (2001), 50-55.

[37] E. Tulving, in: Organization of Memory, E. Tulving and W. Donaldson, eds, Academic, New York, 1972, pp. 381-403.
[38] E. Tulving, Episodic memory: from mind to brain, Аппи Rev Psychol 53 (2002), 1-25.

[39] J.D. Uys, D.J. Stein, W.M. Daniels and B.H. Harvey, Animal models of anxiety disorders, Curr Psychiatry Rep 5 (2003), 274-281.

[40] J.J. Vasterling, K. Brailey, J.I. Constance and P.B. Sutker, Attention and memory dysfunction in posttraumatic stress disorder, Neuropsychology 12 (1998), 125-133.

[41] V. Walsh and A. Cowey, Transcranial magnetic stimulation and cognitive neuroscience, Nat Rev Neurosci 1 (2000), 73 79 . 


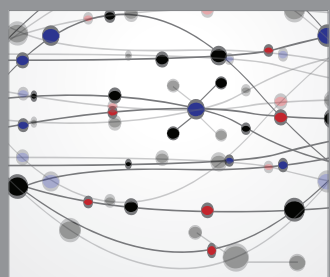

The Scientific World Journal
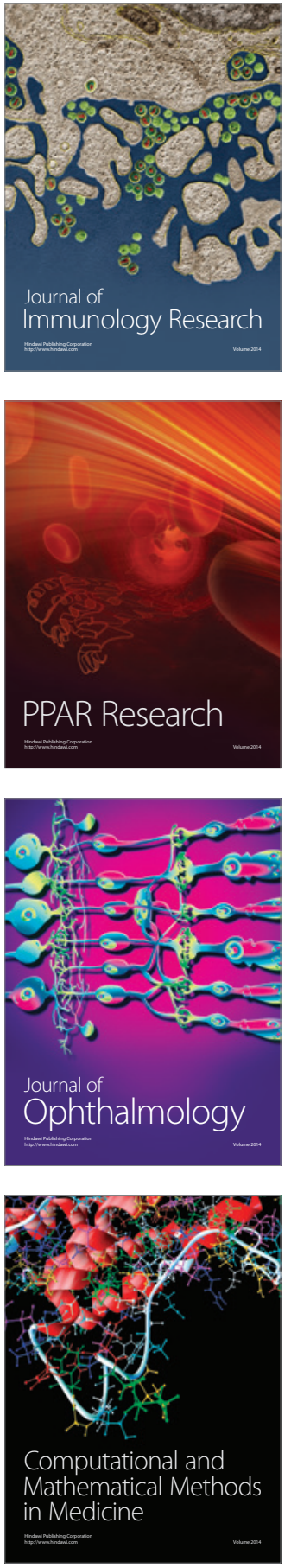

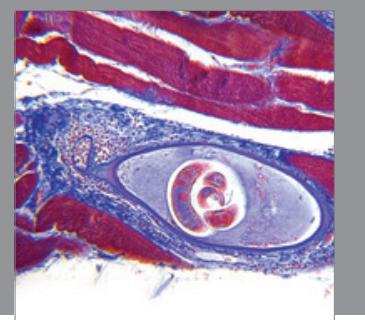

Gastroenterology

Research and Practice
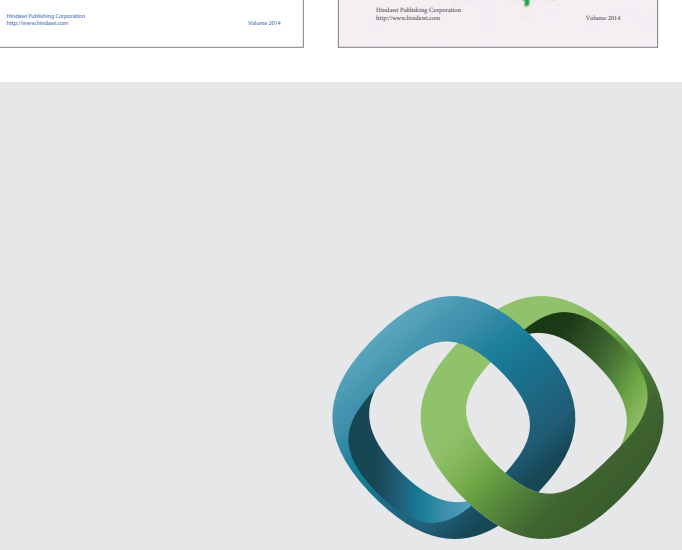

\section{Hindawi}

Submit your manuscripts at

http://www.hindawi.com
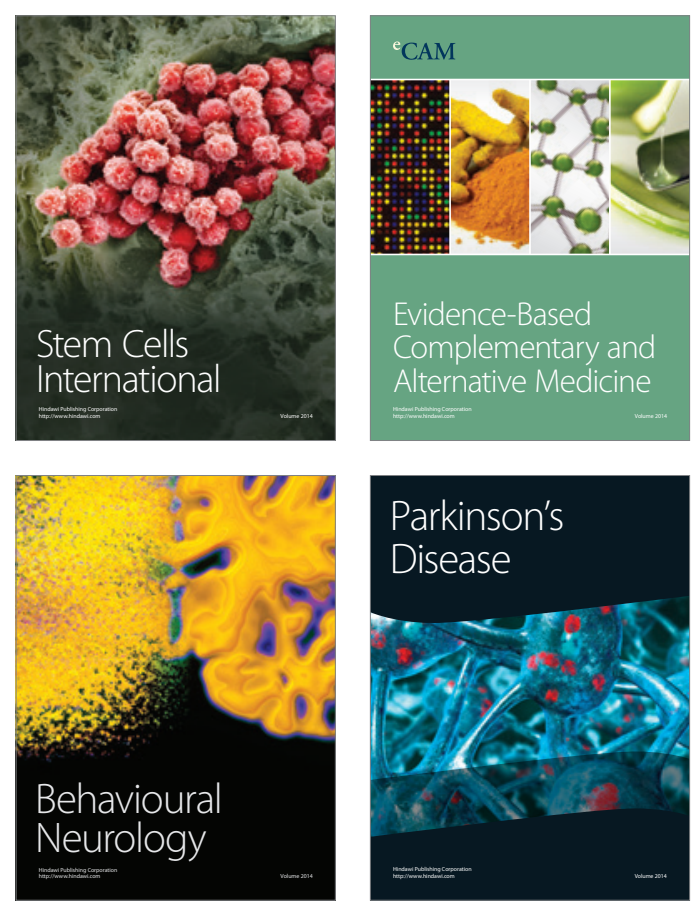

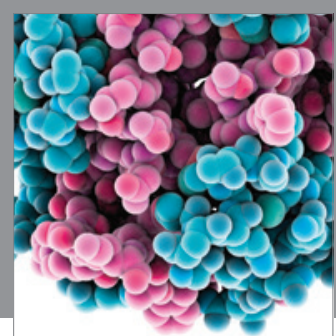

Journal of
Diabetes Research

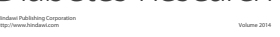

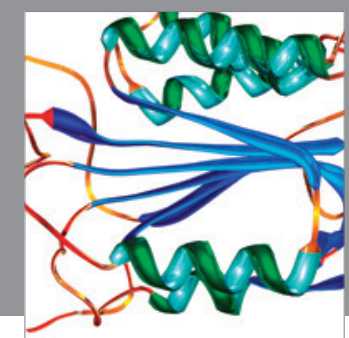

Disease Markers
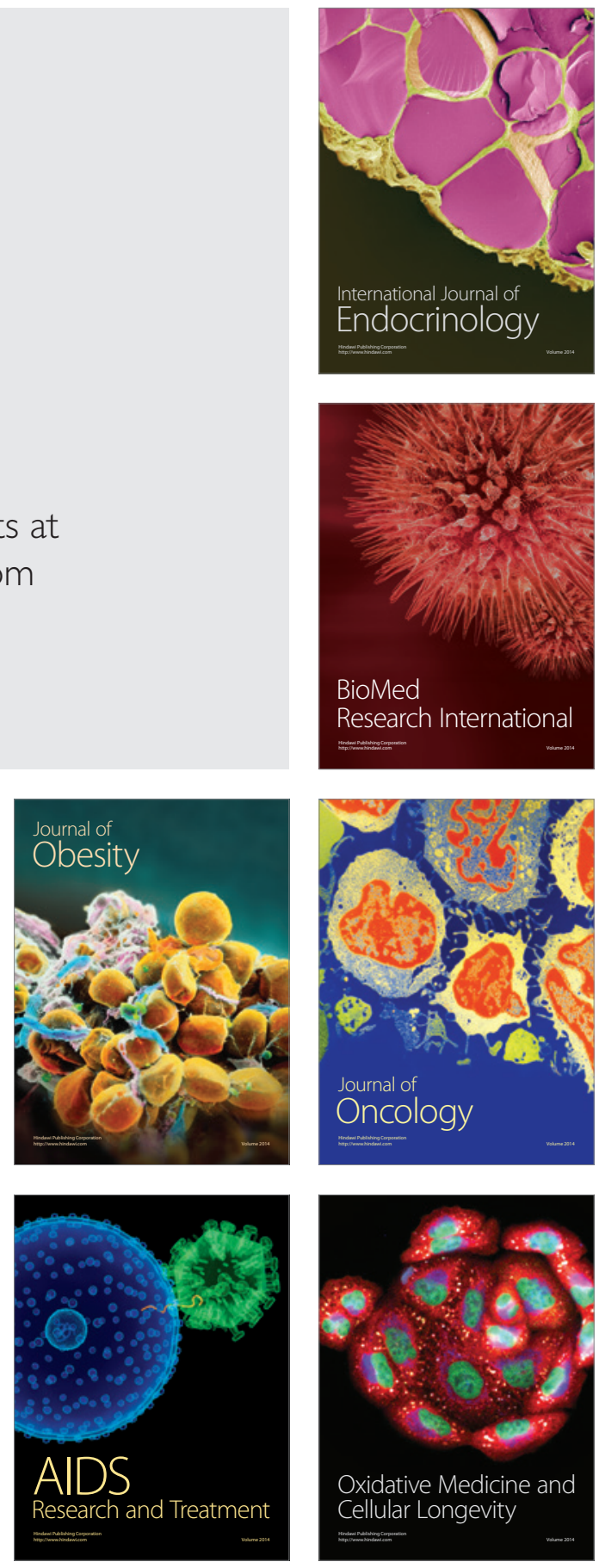